\title{
A Call to Quantify the Impact of KETO Diets
}

\author{
Richard M Fleming ${ }^{1 *}$, Matthew R Fleming ${ }^{1}$, Tapan K Chaudhuri ${ }^{2}$ and Andrew McKusick ${ }^{1,3}$ \\ ${ }^{1}$ FHHI-OmnificImaging-Camelot, Los Angeles, CA, USA \\ ${ }^{2}$ Eastern Virginia Medical School, Norfolk, Virginia \\ ${ }^{3}$ Sebec Consulting and Media, Charlotte, North Carolina \\ *Corresponding Author: Richard M Fleming, Professor, FHHI-OmnificImaging-Camelot, Los Angeles, CA, USA.
}

Received: April 12, 2019; Published: May 21, 2019

DOI: $10.31080 /$ ASPS.2019.03.0289

Keywords: FMTVDM/BEST Imaging ${ }^{\circledR \oplus}$; Breast Cancer; Breast Inflammation; Quantification; AI; Nuclear Camera Quantitative Calibration; Patent protected; Diets; KETO Diets

Are KETO - VEGAN - or OTHER Diets Playing Russian roulette with Your Health?

The First Quantitative Method to Find Heart Disease and Cancer is finally here and Big Pharma, Doctors, Hospitals and DIET PUNDITS are now going to be held accountable!

The ERA of practicing medicine by merely looking at a picture of someone's heart, chest or a mammogram, or taking a patients blood sample to measure cholesterol or inflammation and letting the Doctor decide if they think you have heart disease or cancer is OVER. So too is the ERA of treating someone merely based upon a mammogram, CT, MRI, PET, SPECT/planar imaging or anything else that depends upon physicians deciding yes you have disease or no you don't.

In 2018, CMS, ASNC and the SNMMI all called for improvements in diagnostic testing using the measurement (quantification) of what the computers are seeing, with the endpoint being to reduce clinical errors. No one understands the problems associated with the qualitative visual estimates of disease more than Dr. Richard M. Fleming. Dr. Fleming a Physicist-Physician began working on this problem in 1999, several years after showing that coronary arteriograms, tests frequently used to find narrowing in the arteries of the heart, are frequently misinterpreted resulting in problems with treatment.

His subsequent work lead to his theories on "Inflammation and Heart Disease" and "Angina" resulting in a paradigm shift from simply calling cholesterol the culprit of heart disease to understanding that coronary artery disease is an inflammatory process which builds up in the arteries of the heart making the arteries less likely to relax when needed, to carry more blood when the heart is working harder.
By 1999, Dr. Fleming started working out the problems that were occurring with nuclear imaging cameras and discovered that several companies had misrepresented to the FDA how their drugs worked (FDA-2018-P-3102; FDA-2018-D-4267-0002). He also discovered that the very cameras used to determine what was happening in the heart were not measuring what people thought they were measuring. In fact, the cameras had never been quantitatively calibrated and efforts to make "prettier" pictures were resulting in more information being lost. In the face of this lost information, doctors were being asked to make decisions about whether you had heart disease or not.

When Dr. Fleming began to look at breast cancer, he discovered the same problems existed and he soon discovered that not only were people receiving more radiation than necessary producing a 2-3 fold increase in profits and sales of these isotopes, profiting the pharmaceutical companies, but there were cumulative problems resulting in errors in $20-30 \%$ of the cases.

But all of that changed on Valentines Day 2017. After 18-years of work and investigation, Dr. Fleming was granted the first patent for a truly quantitative artificial intelligence (AI) method for "measuring" heart disease and cancer. The discovery changed the question from do you have disease, to where do you sit on the "Health-Spectrum." Given the ability to measure this "HealthSpectrum" patients are no longer told they have disease, yes/no, but rather where they actually sit on their spectrum of disease. Dr. Fleming finds the use of the term disease, less descriptive of how we should be looking at the problem. This may be a matter of how do you see the glass filled half way to the top with water. Dr. Fleming recognizes that if the glass is half full with water, it is also half full with air. 
While little was known of Dr. Fleming's quantitative work until last year, when he published more than 20-papers in peer reviewed medical journals and presented material to 7-medical conferences, his now well published work is also important for at least three other reasons.

First, the patent called "The Fleming Method for Tissue and Vascular Differentiation and Metabolism (FMTVDM) using same state single or sequential quantification comparisons" (9566037), which includes Breast Enhanced Scintigraphy Test (BEST) Imaging (FMTVDM/BEST) for heart disease and cancer respectively, quantitatively defines where a person is on the transition through the "Health-Spectrum" as shown in figure 1. Secondly, because it can provide an actual measurement of the changes in regional blood flow and metabolism using proprietary equations (Figure 2) developed by Dr. Fleming, when two sequential studies are done the studies can be compared to determine how your health is changing and how rapidly.

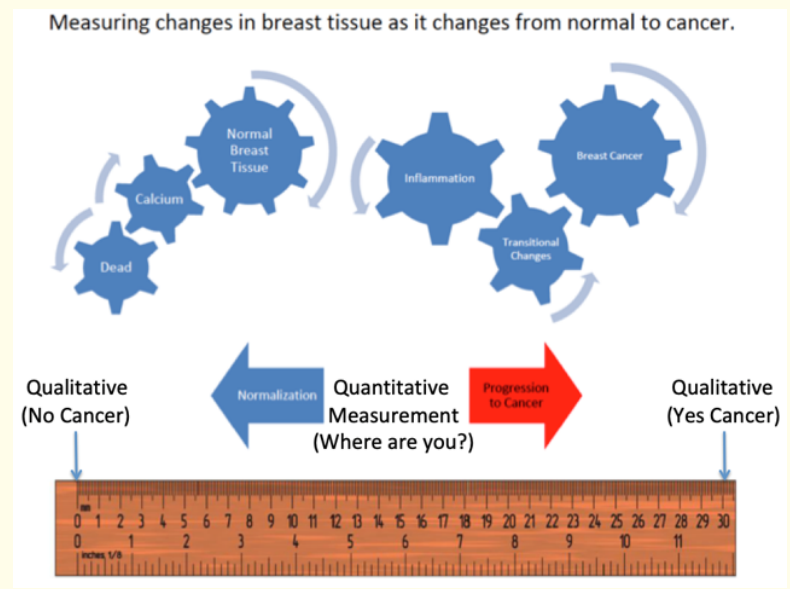

Figure 1: Quantification of the "Health-Spectrum" for Cancer.

\section{FMTVDM ${ }^{\oplus \odot}$ PROTOCOL}

FMTVDMCP "stress-first/stress-only/STRESSSTRESS" imaging sequence was performed in each patient ${ }^{1-5}$, following TFMCP camera calibration.

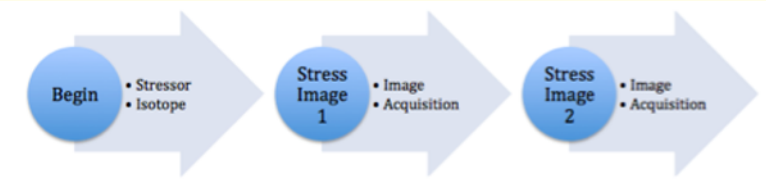

\section{After FMTVDMOP Imaging, acquired images underwent quantification.}

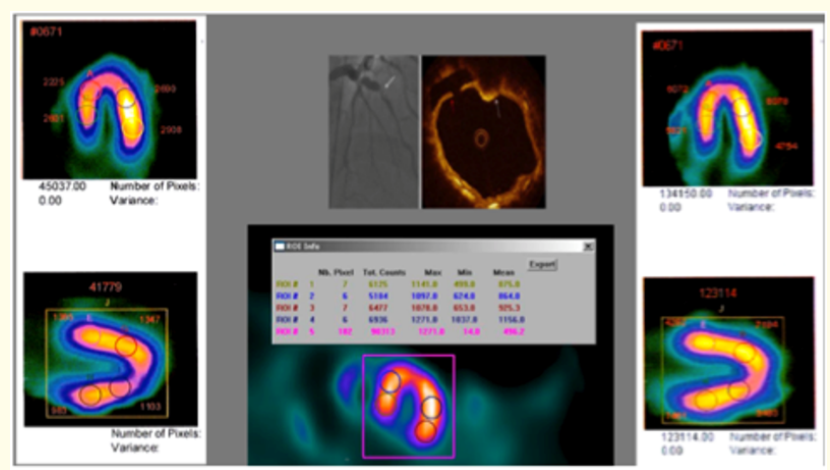

Figure 2: FMTVDM measures critical coronary artery disease missed by qualitative human interpretation. 
Third, FMTVDM/BEST can for the first time using those sequential measurements, measure whether your treatment is working or not; saving time, money and lives. While there are six papers currently out and under embargo, there are three papers already published showing patient-specific, patient-directed treatment outcomes using FMTVDM/BEST for the monitoring and treatment of Breast Inflammation and Breast Cancer. In the most recent paper, the editors agreed,

"Clearly, clinicians now have the ability to quantitatively measure the impact of medical management and treatment be it inter alia diet and lifestyle, medical management, hormonal, immunotherapy, radiation therapy or surgical treatments and they will now be held accountable for quantitative clinical decision making given the availability of this quantitative ability to measure where the patient is on the "Health-Spectrum" and their treatment response."

Given the concerns over the serious potential health consequences of several diets popularized and being used by people today, and Dr. Fleming's studies showing that changes in weight, cholesterol, CRP, homocysteine, interleukin- 6 and other blood tests are not useful for determining if these diets or other treatments are harming people, Dr. Fleming has agreed that he will provide a special license to centers who wish to study the impact of these diets to answer the question: "Are KETO - VEGAN - or OTHER Diets Playing Russian roulette with Your Health?"

Those who believe their DIETS and treatments are beneficial to their patients will welcome the opportunity to prove it, while undoubtedly those less confident of the overall benefit of their recommended DIETS and treatments will be less enthusiastic.

In the mean time, it is fair to say that the entire medical and pharmaceutical industry "has been placed on notice" as they "will now be held accountable" for both the diagnosis and subsequent treatment they provide, absent quantitatively measuring the extent of disease and treatment outcomes using FMTVDM/BEST Imaging.

FMTVDM patent was issued to Dr. Fleming. All figures reproduced by permission

Volume 3 Issue 6 June 2019

(C) All rights are reserved by Richard M Fleming., et al. 